\title{
Quantification of epicardial fat volume using cardiovascular magnetic resonance imaging
}

\author{
Tonye Teme*, Bassel Sayegh, Mushabbar Syed, David Wilber, Lara Bakhos, Mark Rabbat \\ From 17th Annual SCMR Scientific Sessions \\ New Orleans, LA, USA. 16-19 January 2014
}

\section{Background}

A growing body of data has demonstrated a direct relationship between epicardial fat volume(EFV) and cardiovascular diseases. In fact, EFV has been shown to be an independent predictor of coronary atherosclerosis and atrial fibrillation. Currently, there is no standard protocol for assessing EFV using cardiac magnetic resonance imaging (CMR). Quantification of peri-ventricular EFV utilizing end diastolic short-axis cine sequences has been described; however, this technique is often challenging due to inadequate visualization of the pericardium. Oftentimes, the pericardium is better visualized during the systolic phase. Thus, we sought to determine the correlation and reproducibility of conventional EFV quantification using end diastolic with end systolic short-axis slices.

\section{Methods}

We prospectively studied 20 patients with atrial fibrillation (AF) from November 1, 2010 to July 31, 2012 prior to AF ablation who underwent CMR. CMR images were acquired on a 3T scanner (Siemens Trio) by SSFP using a standard short axis stack through the ventricles. Epicardial fat was assessed volumetrically in consecutive short-axis views using the multi-slice method (MSM) during both end-systole and end-diastole (Figure 1). EFV measurements were compared using intra-class correlation coefficients (ICC), Pearson's correlation and Bland-Altman plots. The reproducibility of both EFV techniques was assessed by an additional investigator who was blinded to the results.

\section{Results}

Mean EFV using end-systole were similar to end-diastole $(79.8+/-45.74 \mathrm{ml}$ vs $78.1+/-45.28, \mathrm{p}=0.91)$. End-diastolic

Loyola University Medical Center, Maywood, Illinois, USA epicardial fat volume (EDFV) slightly underestimates endsystolic epicardial fat volume (ESFV) by $2 \%$ (Table 1 ). Absolute difference between EDFV and ESFV was $-1.7 \pm$ $6.0 \mathrm{ml}$. ESFV demonstrates strong correlation and level of agreement with EDFV (Pearson Correlation $=0.99$, $\mathrm{ICC}=0.99$ ). Interobserver variability (kappa) for EDFV was

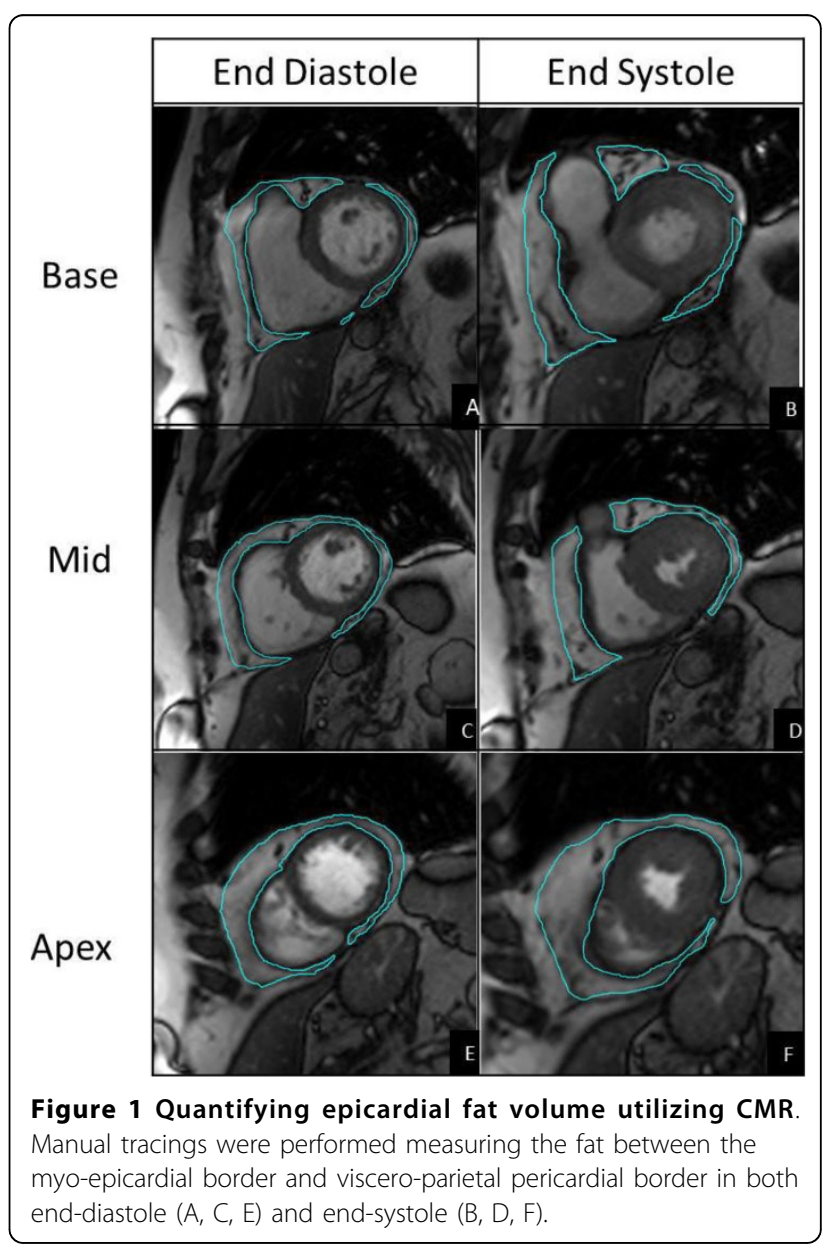


Table 1

\begin{tabular}{cccccc}
\hline $\begin{array}{c}\text { Epicardial Fat } \\
\text { Volume }\end{array}$ & $\begin{array}{c}\text { Absolute } \\
\text { Difference }\end{array}$ & $\begin{array}{c}\text { Percent } \\
\text { Difference }\end{array}$ & ICC & $\begin{array}{c}\text { Pearson } \\
\text { Correlation }\end{array}$ & R2 \\
\hline EDFV vs. ESFV & $-1.7 \pm 6.0$ & $-2.0 \pm 9.7$ & $0.99^{\circ}$ & $0.99^{*}$ & $0.98^{*}$
\end{tabular}

Epicardial fat volume measurements are in $\mathrm{mL}$, Values are presented as means and SD. EDFV = End Diastolic Epicardial Fat Volume. ESFV = End Systolic

Epicardial Fat Volume. ICC = intraclass correlation. R2 = coefficient of

correlation. ${ }^{\circ} 95 \%$ Confidence Interval $=0.98$ to $0.99,{ }^{*}=p$-value $<0.05$.

0.82 (95\% CI: 0.74-0.90) and 0.81 (95\% CI: 0.74-0.88) for ESFV.

\section{Conclusions}

ESFV demonstrates strong correlation, level of agreement and reproducibility with EDFV. When the pericardium is inadequately visualized during the end-diastolic phase, ESFV derived from standard cine sequences offers an alternate method for evaluating epicardial fat with CMR.

\section{Funding}

None.

Published: 16 January 2014

doi:10.1186/1532-429X-16-S1-0112

Cite this article as: Teme et al:: Quantification of epicardial fat volume

using cardiovascular magnetic resonance imaging. Journal of

Cardiovascular Magnetic Resonance 2014 16(Suppl 1):0112. 